\title{
Minimum Leakage Rate (0.5\%) of Stapled Esophagojejunostomy with Sacrifice of a Small Part of the Jejunum after Total Gastrectomy in 390 Consecutive Patients
}

\author{
M. Hyodo Y. Hosoya Y. Hirashima H. Haruta K. Kurashina S. Saito \\ T. Yokoyama W. Arai T. Zuiki Y. Yasuda H. Nagai \\ Department of Surgery, Jichi Medical University, Shimotsuke, Japan
}

Key Words

Esophagojejunostomy $\cdot$ Total gastrectomy $\cdot$ Anastomotic leakage $\cdot$ Stenosis

\begin{abstract}
Background: The development of new surgical instruments and devices has facilitated the performance of esophagojejunostomy after total gastrectomy. However, total prevention of dehiscence of anastomoses remains difficult. We introduced a new procedure for esophagojejunostomy using a circular stapler, requiring sacrifice of only a small part of the jejunum. Methods: The study group comprised 390 consecutive patients who underwent reconstruction by Roux-en-Y esophagojejunostomy, performed with a circular stapler, sacrificing a small part of the jejunum after total gastrectomy. We assessed anastomotic leakage and anastomotic stenosis after surgery. Results: Only 2 patients $(0.5 \%)$ had leakage and $4(1.0 \%)$ had anastomotic stenosis after reconstruction. All the patients were cured by conservative therapy. Conclusions: Esophagojejunostomy performed with a circular stapler after total gastrectomy, with sacrifice of only a small part of the jejunum, is a useful and easy procedure, with a leakage rate of $0.5 \%$.

Copyright $\odot 2007$ S. Karger AG, Basel
\end{abstract}

\section{Introduction}

In recent years, automatic suturing devices have been developed and increasingly used for anastomoses of the gastrointestinal tract. In particular, circular staplers have been used since the 1980s, offering several advantages in terms of safety and feasibility over conventional techniques for esophagojejunostomy after total gastrectomy [1-3]. Especially in Japan, stapled devices are widely used, and stapled esophagojejunostomy has been recognized as the 'gold standard', with a leakage rate of $1 \%$ [4].

Esophagojejunostomy remains critical to the success or failure of reconstruction after total gastrectomy because impairment of this anastomosis can lead to severe postoperative complications, such as peritonitis and subphrenic abscess, prolonging the hospital stay. Esophagojejunostomy is thus an important determinant of the success or failure of total gastrectomy. The development of automatic circular staplers has allowed reconstruction to be accomplished more easily and safely than previously; however, total prevention of dehiscence of anastomoses remains challenging. In this report, we describe our concept and technique for esophagojejunostomy using a circular stapler, with sacrifice of a small part of the jejunum to ensure reliable stapling. We have used this technique in 390 consecutive patients. The leakage rate has been minimal $(0.5 \%)$, with only 2 cases of dehiscence of the esophagojejunostomy.

\section{KARGER \\ Fax +41613061234 E-Mail karger@karger.ch} www.karger.com
Masanobu Hyodo

Department of Surgery, Jichi Medical University

3311-1 Yakushiji

Shimotsuke 329-0498 (Japan)

Tel. +81 28558 7371, Fax +81 28544 3234, E-Mail mhyodo@jichi.ac.jp 

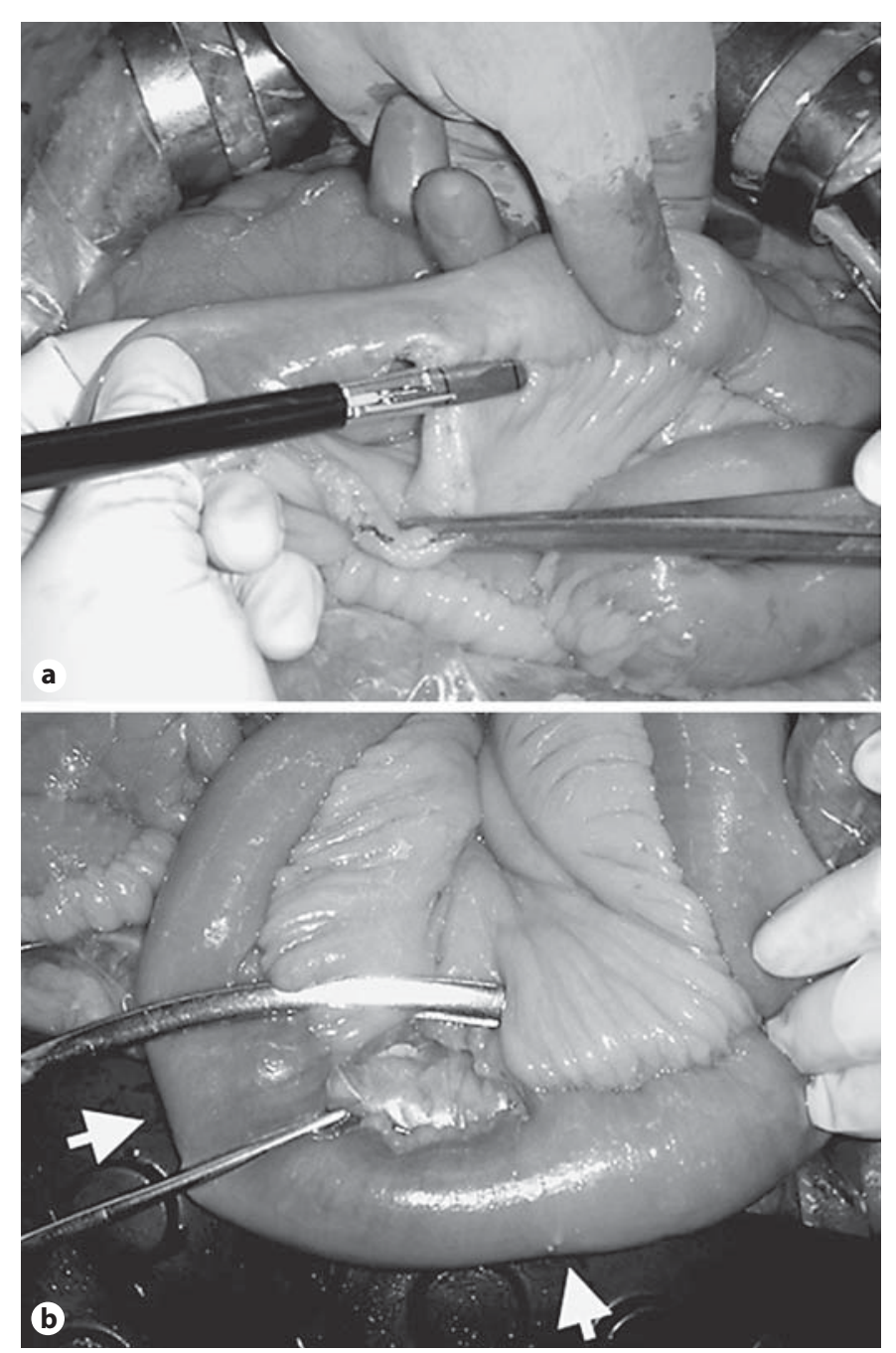

Fig. 1. a The mesentery along the jejunum is dissected with the use of LigaSure (Tyco), preserving the jejunal vascular arcade. b The two white arrows indicate the range of the sacrificed jejunum after dissection of the mesentery.

\section{Methods}

\section{Procedures}

At the time of total gastrectomy, purse-string sutures were placed in the abdominal esophagus with the use of PURSTRING (Tyco, Norwalk, Conn., USA) after adequate dissection of the tissue around the esophagus. The esophageal stump was then grasped with Allis clamps, and the anvil of a circular stapler (PCEEA $25 \mathrm{~mm}$, Tyco) was carefully inserted into the lumen of the esophagus. A thread was tied around the head of the circular stapler, without leaving any slack. The mesentery of the jejunum was then dissected $10-15 \mathrm{~cm}$ lengthwise about $20 \mathrm{~cm}$ distal to the ligament of Treitz; the jejunal vascular arcade was preserved (fig. 1a). The proximal side of the ischemic segment of the jejunum ('sacrificed jejunum') was severed and lifted up to the esoph-
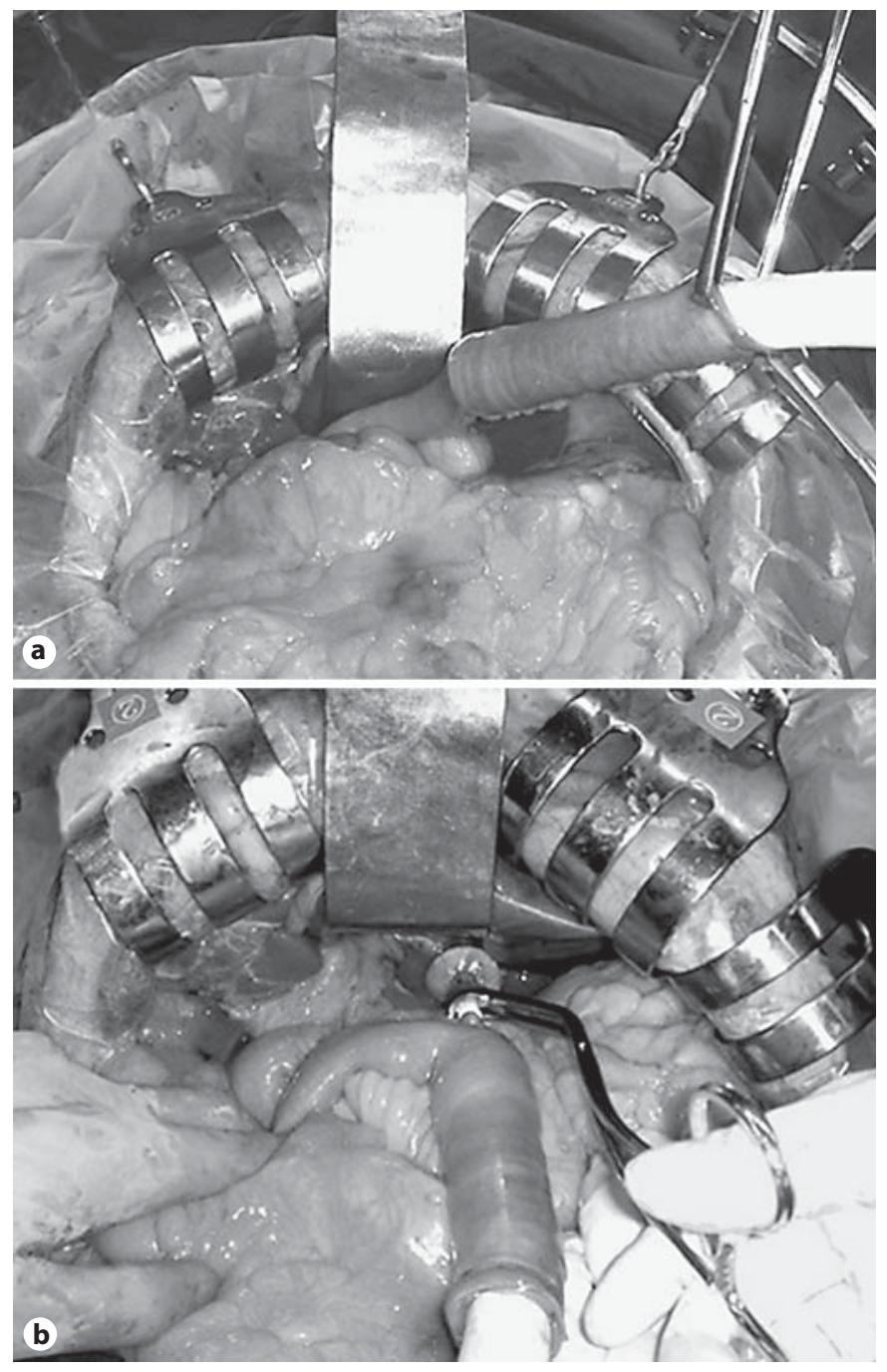

Fig. 2. a The body of the PCEEA (Tyco) is inserted via the sacrificed jejunum. b End-to-side esophagojejunostomy is performed with approximation of the center rod in the jejunum and the anvil in the esophagus.

ageal stump (fig. 1b). The body of the circular stapler was inserted via the sacrificed jejunum (fig. $2 \mathrm{a}$ ) and the viable portion of the jejunum was pierced with the center rod. End-to-side esophagojejunostomy was performed after approximation of the center rod in the jejunum and the anvil in the esophagus (fig. 2b). Finally, the jejunal stump was closed with the use of a linear stapler 3$4 \mathrm{~cm}$ from the esophagojejunostomy and the sacrificed jejunum was removed (fig. 3). For reconstruction, we created a side-toend, Roux-en-Y anastomosis between the esophagus and jejunum by hand sewing, about $40 \mathrm{~cm}$ distal to the esophagojejunostomy.

\section{Patients}

Until 2000, we performed esophagojejunostomy using a circular stapler with dissection of the jenunal arcade without sacrificing any portion of the jejunum. Since 2001, we have performed 


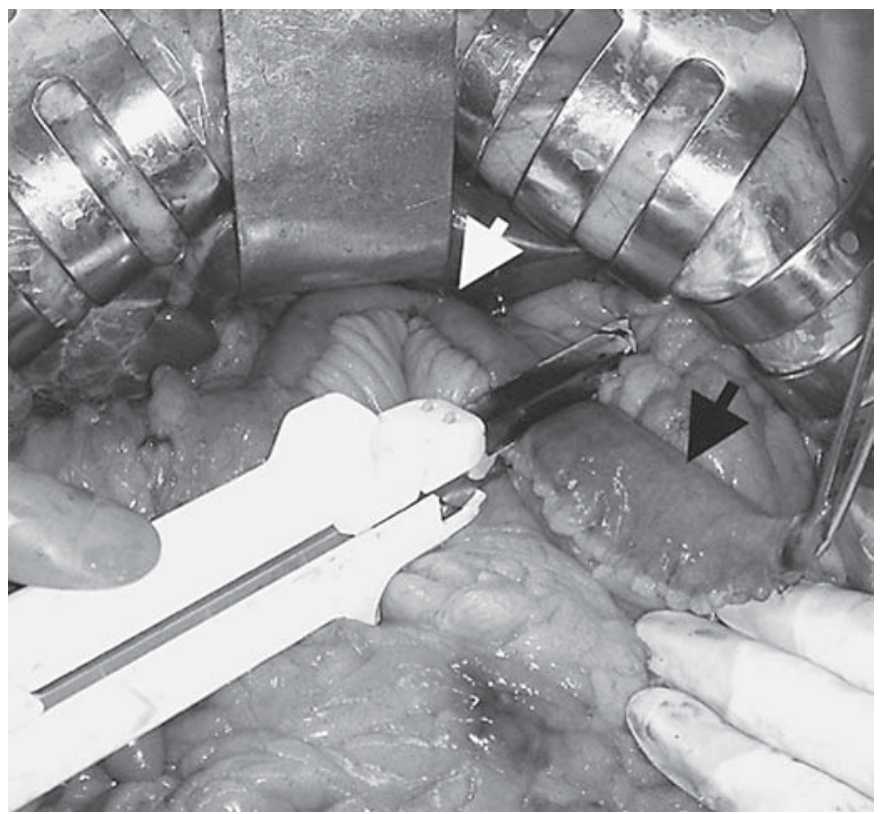

Fig. 3. The sacrificed jejunum is dissected $3-4 \mathrm{~cm}$ from the esophagojejunostomy with a linear stapler (GIA, Tyco). The white arrow indicates the esophagojejunostomy, and the black arrow indicates the sacrificed jejunum.

esophagojejunostomy using a circular stapler with preservation of the jejunal arcade and sacrifice of a small part of the jejunum. This procedure for reconstruction has been done in 390 consecutive patients. We evaluated anastomotic leakage and anastomotic stenosis after surgery. We defined anastomotic leakage as both clinical and nonclinical leakage, as radiologically confirmed after oral administration of contrast medium (grade 1 or higher on CTCAE v3.0) [5]. Anastomotic stenosis was defined as endoscopically confirmed stenosis (grade 1 or higher on CTCAE v3.0).

\section{Results}

Two consultant surgeons supervised all operations. The age of the patients ranged from 21 to 87 (median 68) years. The male/female ratio was $276 / 114$, and the stage of gastric cancer was I in 140 patients, II in 49, III in 93, IV in 101, and other in 7. Splenectomy was concurrently performed in 87 patients. Only 2 patients (0.5\%) had leakage, and 4 (1\%) had anastomotic stenosis. One of the 2 former patients had leakage at the jejunal stump; the other had leakage at the esophagojejunostomy. Both of these patients responded to conservative therapy and were discharged 40 and 28 days after surgery, respectively. All 4 patients with anastomotic stenosis underwent balloon dilatation one or two times 10-34 days after surgery and were discharged 22-43 days after surgery.

Esophagojejunostomy Leakage Rate $0.5 \%$

\section{Discussion}

Esophagojejunostomy is critical to the outcome of total gastrectomy because anastomotic dehiscence can lead to severe complications, such as subphrenic abscess, peritonitis, sepsis, and other life-threatening conditions. The leakage rate of esophagojejunostomy has ranged from 3 to $10 \%$ since the introduction of new devices for anastomosis [1-3]. Even recent studies have not reported a leakage rate of less than $1 \%$ for esophagojejunostomy $[6,7]$. The lowest previously reported leakage rate was $1 \%$ among 588 patients treated at the National Cancer Institute in Japan [4] and 1.35\% among 148 patients in Europe [8]. Our leakage rate of $0.5 \%$ among 390 consecutive patients was extremely low, demonstrating that our procedure for esophagojejunostomy is safe and reliable. Only 2 patients had anastomotic leakage with minor dehiscence, cured by conservative therapy. The rate of anastomotic stenosis of $1.0 \%$ was also very low. Besides these safety advantages, our procedure for performing anastomosis with a circular stapler is simple and expedient.

Our procedure has four other distinct advantages over conventional techniques for esophagojejunostomy. First, because the body of the PCEEA is inserted from the side of the ischemic sacrificed jejunum, which is eventually removed, the portion of the jejunum injured by insertion does not remain. Second, anastomosis can be reliably completed because the body of the PCEEA is held stably by the sacrificed jejunum, without slipping or involving surrounding tissue. Third, dissection of $10-15 \mathrm{~cm}$ of the mesentery along the jejunum creates room for the Rouxen-Y anastomosis, about $40 \mathrm{~cm}$ distal to the esophagojejunostomy. Fourth, blood flow to the anastomosis may be maintained because the jejunal vascular arcade is preserved.

Although our procedure is simple, several precautions are needed. The entire layer of the esophagus must be secured around the anvil head of the PCEEA. The esophagus should be dissected 2 or $3 \mathrm{~cm}$ proximally to the resection line, and the thread of PURSTRING should be tied tightly around the esophagus, leaving no slack. The most important point is the degree of tension between the esophagus and jejunum. Our procedure is occasionally contraindicated for patients undergoing combined resection of the lower esophagus because too much tension is created when the jejunum with the vascular arcade is raised to the mediastinum. We usually check that the tension of the jejunum is not excessive when the site of the anastmosis is raised to the esophageal stump via the antecolic route, which is longer than the retrocolic one. As

Dig Surg 2007;24:169-172 
for the use of the PCEEA, we squeeze the knob without haste, maintaining an equal balance between the esophageal side and jejunal side. At the same time, the operator should carefully protect the anastomotic site and not unnecessarily involve adjacent tissue, such as the mesentery or opposite side of the jejunum.

We think that our very low leakage rate of $0.5 \%$ for stapled esophagojejunostomy involves a learning curve. Nomura et al. [4] recommended that the technique of esophagojejunostomy should be evaluated in the light of a learning curve. The leakage rate of 5\% among the 100 patients undergoing esophagojejunostomy at our hospital from 1999 to 2000 (data not shown) was higher than that reported by Nomura et al. [4]. The dramatic decrease in leakage was attributed to the learning curve in our 2 consultant surgeons.

Another important aspect of esophagojejunostomy after total gastrectomy is the cost involved. The extremely low leakage rate with our procedure apparently contributed to reduced medical costs after surgery. A good hospital course after total gastrectomy, a short hospital stay, and no need for special treatment for leakage are also likely to lower medical costs. Although a circular stapler and a linear stapler must be used for esophagojejunostomy and closure of the jejunal stump, respectively, we believe that the use of these instruments is reasonable in terms of expediency and outcomes.

The development of new instruments for gastrointestinal anastomoses has facilitated the performance of esophagojejunostomy after total gastrectomy. Our method for esophagojejunostomy using a circular stapler with sacrifice of a small part of the jejunum is expected to make esophagojejunostomy much easier and safer, with a leakage rate of $0.5 \%$.

\section{References}

1 Walter BS, Oscarson JE, Graffner HO, Vallgren S, Evander A: Esophagojejunostomy with the EEA staple. Surgery 1986;99:598603.

-2 Kataoka M, Masaoka A, Hayashi S, Honda H, Hotta T, Niwa T, Honda K: Problems associated with the EEA stapling technique for esophagojejunostomy after total gastrectomy. Ann Surg 1989;209:99-104.

-3 Habu H, Kando F, Saito N, Sato Y, Takeshita K, Sunagawa M, Endo M: Experience with the EEA stapler for esophagojejunostomy. Int Surg 1989;74:73-76.
-4 Nomura S, Sasako M, Katai H, Sano T, Maruyama K: Decreasing complication rates with stapled esophagojejunostomy following a learning curve. Gastric Cancer 2000;3:97101.

5 Common Terminology Criteria for Adverse Events v3.0 (CTCAE v3.0). http://ctep.cancer.gov/forms/CTCAEv3.pdf.
Ichikawa D, Kurioka H, Yamaguchi T, Koike H, Okamoto K, Otsuji E, Shirono K, Shioaki Y, Ikeda E, Mutoh F, Yamagishi H: Postoperative complications following gastrectomy for gastric cancer during the last decade. Hepatogastroenterology 2004;51:613-617.

7 Lamg PJ, Griffin SM, Chandrashekar MV, Richardson DL, Karat D, Hayes N: Prospective study of routine contrast radiology after total gastrectomy. Br J Surg 2004;91:10151019.

8 Pesko P, Bjelovic M, Stojakov D, Sabljak P: Modified stapling technique of esophagojejunal anastomosis. Dis Esophagus 2002;15: 303-304. 